\title{
Valoración funcional en pacientes intervenidos mediante plastia de LCA con semitendinoso-recto interno y sistema Togglelock. \\ DOI: http//dx.doi.org/10.37315/SOTOCAV202028355108
}

\author{
DESCALZO-PÉREZ $P^{1}$, MIFSUT-MIEDES $D^{1,2}$, RODRÍGUEZ-COLLELL JR², LóPEZ-SÁNCHEZ $A^{2}$, SILVESTRE- \\ MUÑOZ A ${ }^{1,2}$.
}

1. DEPARTAMENTO DE CIRUGÍA. UNIVERSITAT DE VALÈNCIA.

2. SERVICIO DE CIRUGÍA ORTOPÉDICA Y TRAUMATOLOGÍA. DEPARTAMENTO DE SALUD DE VALENCIA CLÍNICOMALVARROSA

\section{Resumen.}

Introducción: Una de las lesiones más frecuente de la rodilla y que está en aumento, sobre todo en el ámbito deportivo es la lesión del ligamento cruzado anterior. La reconstrucción quirúrgica es fundamental para recuperar la biomecánica de la rodilla, proporcionar una correcta estabilidad y funcionamiento libre de dolor, así como evitar cambios degenerativos tempranos. El objetivo del presente estudio fue valorar la recuperación funcional de los pacientes sometidos a una reconstrucción del ligamento cruzado anterior mediante ligamentoplastia con tendón semitendinoso-recto interno y sistema Toggelock. Material y métodos: Se diseñó un estudio retrospectivo descriptivo observacional, en el que se incluyeron a 29 pacientes a los cuales se les realizó una ligamentoplastia con semitendinoso-recto interno y sistema Togglelock en nuestro Centro, durante los años 2017-2018. Se estudiaron variables obtenidas de la historia clínica y la escala de Lysholm para la valoración del paciente. Resultados: En total, se obtuvo una muestra de 29 pacientes, 26 hombres y 3 mujeres con una media de edad de 31,13 años (rango 19-53). La rodilla más frecuentemente lesionada fue la derecha en 17 pacientes de 29, presentando lesiones asociadas el $65 \%$. Una paciente había sufrido una fractura tibial intervenida con un clavo endomedular que alteró los resultados finales. La puntuación postquirúrgica de la escala de valoración subjetiva de Lysholm fue de 86. La movilidad de la rodilla intervenida presentó una reducción mínima de flexión de $6^{\circ}$ en comparación con la rodilla no operada estadísticamente significativa. Conclusión: Los resultados obtenidos tras la reconstrucción del LCA con dicha técnica, utilizando la plastia del semitendinoso-recto interno y sistema Togglelock fueron buenos-excelentes, con un alto grado de satisfacción en los pacientes, escasas complicaciones y con resultados funcionales notables.

Palabras clave: Rodilla. Ligamento cruzado anterior. Reconstrucción Togglelock, Ligamentoplastia. Artroscopia. Valoración funcional.

\footnotetext{
Summary.

Introduction: One of the most frequent knee injuries and one that is increasing, especially in sports, is the anterior cruciate ligament injury. Surgical reconstruction is essential to recover the biomechanics of the knee, provide correct stability and pain-free function, as well as avoid early degenerative changes. The objective of the present study was to assess the functional recovery of patients undergoing reconstruction of the anterior cruciate ligament by means of ligamentoplasty with semitendinosus- internal rectus tendon and Toggelock system. Material and methods: A retrospective descriptive observational study was designed, which included 29 patients who underwent a ligamentoplasty with internal semitendinosusrectum and Togglelock system in our Center, during the years 2017-2018. Variables obtained from the clinical history and the Lysholm scale were studied to assess the patient. Results: In total, a sample of 29 patients was obtained, 26 men and 3 women with a mean age of 31.13 years (range 19-53). The most frequently injured knee was the right one in 17 patients out of 29 , with $65 \%$ presenting associated injuries. One patient had suffered a tibial fracture operated with an intramedullary nail that altered the final results. The postsurgical score on the Lysholm subjective assessment scale was 86 . The mobility of the operated knee showed a minimal reduction in flexion of $6^{\circ}$ compared to the non-operated knee that was statistically significant. Conclusion: The results obtained after ACL reconstruction with this technique, using the semitendinosus- internal rectum plasty and the Togglelock system were good-excellent, with a high degree of patient satisfaction, few complications, and notable functional results.
} 


\section{Correspondencia:}

Damián Mifsut Miedes

mifsut.dam@gmail.com

\section{Introducción}

La incidencia de las de roturas del Ligamento Cruzado Anterior (LCA) ha ido creciendo debido al aumento de la participación de la población en actividades deportivas. Se calcula que al año 1 de cada 3.000 personas sufren una ruptura del $\mathrm{LCA}^{1}$. Se considera la lesión más común $(50 \%)$ dentro de todas las lesiones de rodilla, y representa aproximadamente el $60 \%$ de las cirugías relacionadas con el deporte; esto supone unas 100.000 lesiones de este tipo anuales y un gasto de unos 2.000 millones en su reconstrucción y posterior rehabilitación en Estados Unidos ${ }^{2}$.

La incidencia aumenta en los deportes donde hay contacto y en aquellos deportes donde se realicen movimientos de pivotaje, cambios de dirección, donde haya saltos, rotaciones internas como es el caso del futbol, baloncesto, balonmano y esquí ${ }^{3}$.

La inestabilidad residual resultante de esta lesión altera la biomecánica normal de la rodilla y predispone al desarrollo de cambios degenerativos precoces en dicha articulación.

El manejo de este tipo de lesiones es considerado actualmente quirúrgico, para mejorar la estabilidad de la rodilla y reducir la incidencia de desgarros meniscales posteriores, así como disminuir la probabilidad de presentar datos radiológicos de degeneración articular.

Los objetivos del presente trabajo fueron:
1. Evaluar la recuperación funcional de los pacientes con rotura traumática del ligamento cruzado anterior a los que se les realiza una ligamentoplastia mediante la técnica de plastia de semitendinoso-recto interno (monofascículo) y sistema Togglelock, mediante la escala de Lysholm.

2. Analizar las características de los pacientes sometidos a esta técnica por rotura traumática del LCA.

3. Evaluar la estabilidad de la rodilla en el examen final previo al alta.

4. Evaluar la seguridad de la técnica, y sus complicaciones.

\section{MATERIAL Y MÉTODOS}

Se llevó a cabo un estudio restrospectivo descriptivo observacional de una serie de 29 casos de pacientes intervenidos en nuestro Centro por rotura del LCA, entre los años 2017-2018, mediante la técnica de reconstrucción monofascicular con semitendinoso-recto interno con tenosuspensión Togglelock y tornillo biodegradable tibial. El tiempo de seguimiento mínimo fue de un año.

De las historias clínica se recabaron las siguiente variables: edad, género, rodilla lesionada, mecanismo de lesión, antecedentes quirúrgicos en la rodilla, comorbilidades, lesiones meniscales asociadas, test funcionales (Lachman y cajón anterior) y de valoración subjetiva de la rodilla mediante la escala de Lysholm $^{4}$ (Fig. 1), la movilidad, dolor o bloqueos y las posibles complicaciones. Todos los pacientes intervenidos fueron citados a la consulta para su evaluación.

\begin{tabular}{|c|c|c|c|c|c|c|c|}
\hline \multicolumn{2}{|l|}{ Inestabilidad } & \multicolumn{2}{|l|}{ Dolor } & \multicolumn{2}{|l|}{ Bloqueo } & \multicolumn{2}{|l|}{ Hinchazón } \\
\hline Nunca & 25 & Nunca & 25 & Nunca & 15 & Nunca & 10 \\
\hline Durante e raras veces & 20 & Moder., durante el ejercicio & 20 & Enganche sin bloqueo & 10 & Durante ejercicios intensos & 6 \\
\hline Durante el ejer.. con frecuencia & 15 & Fuerte, durante el ejercicio & 15 & Bloqueo ocasional & 6 & Durante actividad cotidiana & 2 \\
\hline Ocasional, vida cotidiana & 10 & Marcha $>2 \mathrm{~km}$, fuerte & 10 & Bloqueo frecuente & 5 & Constante & 0 \\
\hline Frecuente, vida cdtidiana & 5 & Marcha -2 Sen, fuerte & 5 & Bloqueo agudo dar examen & 2 & & \\
\hline Acada paso & o & Constante & 0 & & & & \\
\hline \multicolumn{2}{|l|}{ Escaleras } & \multicolumn{2}{|l|}{ Cuclillas } & \multicolumn{2}{|l|}{ Cojera } & \multicolumn{2}{|l|}{ Bastón } \\
\hline Ninguna molestia & 10 & Ningna molestia & 5 & No & 5 & Nunca & 5 \\
\hline Leve dificultad & 6 & Li Leve dificultad & 4 & Moderada u ocasional & 3 & Siempre & 2 \\
\hline De a un escalás & 2 & Li No más de 90 & 2 & Intensa y constante & 0 & Estacion bipeda imposible & 0 \\
\hline Imposible & 0 & Li Imposible & 0 & & & & \\
\hline Lysholm TOTAL: ........... & & $\begin{array}{c}0 \text { a } 64 \text { puntos } \\
\text { MALO }\end{array}$ & & $\begin{array}{l}65 \text { a } 83 \text { puntos } \\
\text { MEDIANO }\end{array}$ & & $\begin{array}{c}84 \text { a } 100 \text { puntos } \\
\text { BUENO/EXCELENT }\end{array}$ & \\
\hline
\end{tabular}

Figura 1: Escala de Lysholm 
Para el análisis de los datos obtenidos del estudio se utilizó el Statistical Package for the Social Science versión 25. Para evaluar la normalidad se utilizó el test estadístico de Shapiro-Wilk. En la mayoría de las variables se utilizaron descriptivos de frecuencia. Para evaluar la movilidad se utilizó el test de Wilkoxon, y para valorar la posible correlación entre lesiones asociadas y la escala de Lysholm se utilizó el test Tau b de Kendall.

La técnica quirúrgica utilizada fue monotunel tibial y femoral y plastia de semitendinoso-recto interno con sistema de tenosuspensión de ToggleLok (Fig. 2) con tecnología ZipLoop y fijación tibial con tornillo biodegradable.

La recopilación de los casos fue totalmente voluntaria por parte de los pacientes, tras su consentimiento informado y aceptación de la inclusión en el estudio. Los datos estuvieron exentos de filiación y no contienen información personal ni confidencial de los pacientes ni de sus familiares.

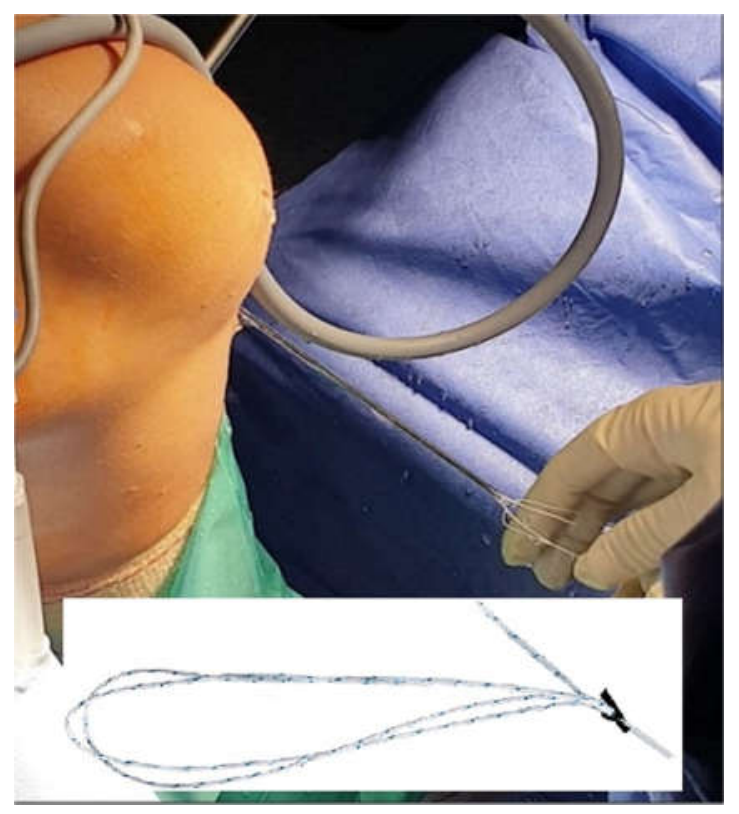

Figura 2: Sistema ToggleLok

\section{RESULTADOS}

La muestra tuvo un tamaño de 29 pacientes, de los cuales 26 fueron hombres (90\%) y 3 mujeres $(10 \%)$. La edad en el momento de la intervención (2017-18), oscilaba entre un mínimo de 19 años y una edad máxima de 53 , con una media de 31,14 años (DT = 10.47; EEM = 1.94) (Fig. 3) y un IC para la media al $95 \%$ de $27,16-35,12$. En cuanto a la rodilla lesionada, 17 fueron derechas (59\%) y 12 izquierdas (41\%). La distribución de los datos en las variables cuantitativas no se ajustó a la normalidad (prueba de Shapiro-Wilk).

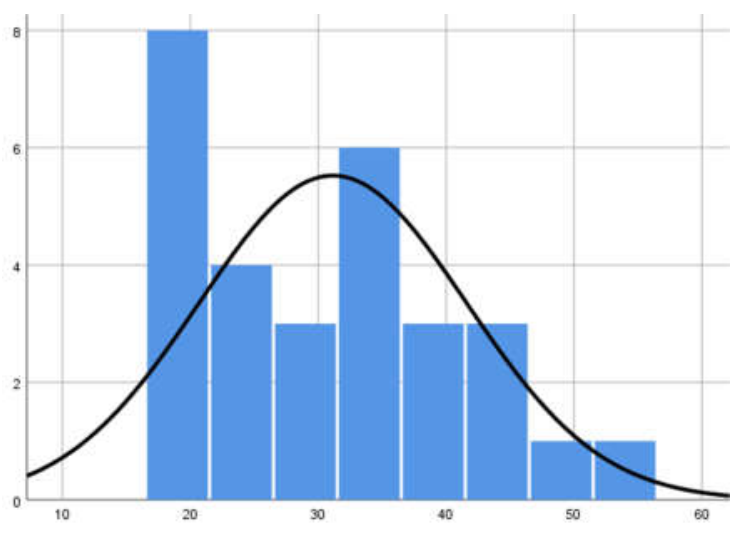

Figura 3: Histograma para la variable Edad

En cuanto a los antecedentes médicos, solo 8 pacientes presentaban alguna enfermedad médica y respecto a los antecedentes quirúrgicos en la misma rodilla, un paciente (3\%) había sido intervenido de ambos meniscos y otro paciente $(3 \%)$ había sido operado del menisco interno con anterioridad. Uno de los pacientes $(3 \%)$ había sufrido una fractura diafisaria de tibia por lo que había sido intervenido mediante un enclavado endomedular y posterior retirada del material de osteosíntesis.

La causa más frecuente de lesión fue la Casual con 9 pacientes $(31 \%)$, la segunda fue el fútbol, con 7 casos (24\%), por último, los accidentes de tráfico sólo estuvieron representados por dos pacientes $(7 \%)$ al igual que el esquí y el baloncesto.

En el estudio preoperatorio recogido en la historia clínica, 23 de los pacientes estudiados (79\%) presentaban inestabilidad tanto en la prueba de Lachman y en el cajón anterior. El resto, es decir, 6 $(21 \%)$ de los pacientes el resultado fue negativo. En cuanto al dolor, estuvo presente en 21 pacientes (72\%).

El tiempo de seguimiento medio fue de 15 meses, mínimo de un año.

De los 29 pacientes estudiados, en el momento de la reconstrucción, $19(65 \%)$ recibieron tratamiento simultáneamente por roturas meniscales asociadas (meniscectomía parcial o subtotal). En concreto, 5 pacientes $(17 \%)$ presentaban lesiones asociadas en menisco externo (ME) y $11(38 \%)$ en menisco interno (MI). Uno de los pacientes (3\%) mostró lesión asociada en $\mathrm{Ml}$ y gonartrosis, simultáneamente, y otros dos $(7 \%)$ presentaban lesiones en ambos meniscos. Por último, dos pacientes presentaron gonartrosis $(7 \%)$ y uno $(3 \%)$ lesión condral.

En la revisión especifica realizada para este estudio en consultas externas, se evaluó la pérdida de movilidad comparando la rodilla intervenida, en flexión y extensión, con la rodilla sana. Dado que, como se verificó previamente, los datos no se ajustaban a la 
normalidad, para realizar las comparaciones se aplicó la prueba no paramétrica (o de libre distribución) de Wilcoxon (equivalente a la $t$ de Student para muestras relacionadas). Pese a que dicha prueba realiza el cálculo mediante rangos, para mayor claridad, a continuación, se aporta la tabla de estadísticos básicos para ambas rodillas en flexión y extensión (Tabla I).

\begin{tabular}{|c|c|c|c|}
\hline & & Media & DT \\
\hline \multirow{2}{*}{$\begin{array}{l}\text { Par } \\
1\end{array}$} & Rango RS flex & 133,57 & 6,215 \\
\hline & Rango RO flex & 126,96 & 6,574 \\
\hline \multirow{2}{*}{$\begin{array}{l}\text { Par } \\
2\end{array}$} & Rango RS Ext & 1,96 & 3,930 \\
\hline & Rango RO Ext & 1,61 & 4,524 \\
\hline
\end{tabular}

Tabla I:. Estadístico para muestras emparejadas del rango de movilidad en rodilla intervenida respecto a la sana.

Los resultados de la prueba de Wilcoxon mostraron diferencias estadísticamente significativas en el caso de la flexión, $-3.89^{\circ}(p<.001)$. En el caso de la extensión, no se detectaron diferencias estadísticamente significativas.

La puntuación media en el test de Lysholm fue de 86 puntos (es decir, dentro del rango entre buena y excelente) con un rango de puntuaciones entre un mínimo de 27 y un máximo de 100. Considerando la variable a nivel categórico (Bueno-excelente: 84-100; Moderado: 65-83; Malo: 0-64), los resultados indican (ver tabla II) que cuatro pacientes se situaron en la categoría de "moderado" y 1 , en la de "Malo", el resto obtuvo resultados buenos-excelentes (Tabla II).

\begin{tabular}{|c|c|c|c|}
\hline & & Frecuencia & Porcentaje \\
\hline \multirow[t]{4}{*}{ Válido } & Malo & 1 & 3,4 \\
\hline & Moderado & 4 & 13,7 \\
\hline & Excelente & 24 & 82,7 \\
\hline & Total & 29 & 100,0 \\
\hline
\end{tabular}

Tabla II: Agrupación de resultados según las categorías : Bueno-excelente: 84-100; Moderado: 65-83; Malo: 064 en el test de Lysholm.

No se encontró correlación entre la presencia de lesiones asociadas y los resultados obtenidos en la escala de Lysholm.
No se observaron complicaciones postquirúrgicas, y solo un caso presentó una rotura de la plastia después de 11 meses de la cirugía. Una de las secuelas que se repetía en la mayoría de los pacientes $24 / 29$ casos $(82,7)$, fue la limitación de los últimos grados de flexión de la rodilla en bipedestación contra gravedad, pero esta mínima limitación no era percibida por el paciente ni suponía ninguna repercusión funcional.

\section{DISCUSIÓN}

La reconstrucción del ligamento cruzado anterior trata de devolver la normalidad anatómica y funcional a la rodilla mediante la utilización de plastias tendinosas. Pero la reconstrucción anatómica exacta viene dificultada tanto por el instrumental como por las plastias utilizadas. Con este objetivo, se han empleado diferentes técnicas, monofasciculares, bifasciculares con doble túnel femoral y tibial, o doble femoral y único tibial, etc., con mayor o menor dificultad técnica, aunque con resultados clínicos y funcionales similares ${ }^{5-7}$.

Considerando además la gran variabilidad anatómica de las áreas de inserción del LCA, en el fémur, el rango de esta área presenta unos valores variables desde 83 hasta 196,8 mm2, según los autores. La superficie de inserción del LCA en la tibia también presenta un amplio rango de valores que varían desde 114 hasta $229 \mathrm{~mm} 2$ según los estudios. Cada una de estas áreas, se divide en dos sectores de inserción para el fascículo anteromedial y posterolateral, respectivamente. Esta división sectorial en fascículos también presenta proporciones diferentes según los estudios anatómicos ${ }^{8,9,10-14}$.

El nuestro trabajo observamos unos buenos resultados funcionales y un alto índice de satisfacción de los pacientes, para la escala Lysholm la media fue de 86 , lo que equivale según la categorización a un resultado entre bueno y excelente. Una media similar obtuvo Palmieri Bouchan et al. ${ }^{15}$ con un 87,8 , Jaramillo Quiceno et al. ${ }^{16}$ con un 85,5 y García et al. ${ }^{17}$ con un 83,77 . En cambio, en otros estudios obtuvieron resultados superiores que en el nuestro entre $90-98^{18-20}$. Estudiamos si los resultados que obtuvimos mediante la escala de Lysholm se explicaban por la presencia de lesiones asociadas junto a la lesión del LCA, pero no se encontraron diferencias, por lo que no presentaban correlación.

En cuanto a la movilidad, se observó una pérdida mínima de flexión en la rodilla lesionada de $6^{\circ}$ respecto a la rodilla sana. En el estudio de Beynnon et al. ${ }^{21}$ los resultados fueron similares. Hubo una pérdida de flexión de $6^{\circ}$ en el caso de los pacientes que fueron intervenidos con injerto del cuádriceps, y de $7^{\circ}$ en el grupo de pacientes intervenidos con injerto de los isquiotibiales. Gigante ${ }^{19}$, al igual que en nuestro caso, no hubo diferencias en la extensión de la rodilla. En 
cambio, en los resultados de Vilchez-Cavazos et al. ${ }^{20}$ fue al contrario. No hubo diferencias en cuanto a la flexión de la rodilla, pero sí que las hubo en la extensión.

La lesión meniscal asociada a la lesión del ligamento cruzado anterior está presente en la mayoría de los estudios con un porcentaje alto. En nuestro estudio se presentó en un $65 \%$, resultado muy similar al que obtuvo el Arroyo-Aparicio et al. ${ }^{18}$ con un $64,4 \%$; el estudio de Palmieri Bouchan et al. en $2018^{15}$ reporta un $79 \%$ y García et al. ${ }^{17}$ un $76,9 \%$. La mayoría de nuestros pacientes, el menisco que con mayor frecuencia se afectó fue el menisco medial. Dicho menisco también fue predominante en el estudio de García et al. ${ }^{17}$ con un $30,8 \%$, sin embargo, en el resto, fue el menisco lateral el más dañado, con un $37 \%$ en los resultados recogidos por Palmieri Bouchan et al. ${ }^{15}$ y un $53 \%$ según VilchezCavazos et al. $^{20}$ en el 2019. La lesión de ambos meniscos se encontró presente en los estudios de Palmieri Bouchan et al. ${ }^{15}$ y de García et al. ${ }^{17}$ con un $30 \%$ y $26,9 \%$ respectivamente, resultados muy superiores en comparación con los nuestros.

La lesión condral asociada a la rotura del LCA, solo lo presentó García et al. ${ }^{17}$ con un $38,5 \%$, porcentaje muy elevado con respecto a nuestro estudio que solo se halló en un paciente.

A la hora de realizar este estudio nos hemos encontrado con algunas limitaciones. Primero, al tratarse de un estudio de diseño retrospectivo, limitó la obtención de datos registrados en la historia clínica. Segundo, las conclusiones a las que podemos llegar con esta investigación son limitadas al tratarse de un estudio con un tamaño muestral reducido, heterogéneo y no controlado. Tercero, no fue posible comparar las puntuaciones preoperatorias de la escala de valoración de Lysholm, ya que no fueron registradas previa a la cirugía.

\section{CONCLUSIONES}

La técnica de plastia con semitendinoso-recto interno y sutura Togglelock es una técnica adecuada con la que se obtiene unos buenos-excelentes resultados en la mayoría de los casos, con un índice de funcionabilidad alto y bajo índice de ruptura.

La mayoría de los pacientes sometidos a la ligamentoplastia son jóvenes varones, deportistas con afectación de la rodilla derecha y con presencia de lesiones asociadas, habitualmente meniscopatías.

Con la técnica utilizada se consigue la estabilidad de la rodilla, el correcto funcionamiento libre de dolor, y mejorar la biomecánica de la rodilla.

Se trata de una técnica segura, reproducible y con escasa morbilidad en la zona donante. 


\section{BIBLIOGRAFÍA}

1. Arabia JJM, Arabia WHM. Lesiones del ligamento cruzado anterior de la rodilla. latreia 2009; 22(3):256-71.

2. Kaeding CC, Léger-St-Jean B, Magnussen RA. Epidemiology and Diagnosis of Anterior Cruciate Ligament Injuries. Clin Sports Med $2017 ; 36(1): 1-8$.

3. Moraleda BR, Cuellar Á, González J, Bastida N, Echarri E, Gallardo J, et al. Revisión de los factores de riesgo y los programas de prevención de la lesión del ligamento cruzado anterior en fútbol femenino: propuesta de prevención. [Review risk factors and prevention programs of the anterior cruciate ligament injury in female football: prevention proposal]. RICYDE Rev Int Cienc Deporte 2017; 13(48):117-38.

4. Lysholm J, Gillquist J. Evaluation of knee ligament surgery results with special emphasis on use of a scoring scale. AmJ Sport Med $1982 ; 10: 150-4$.

5. Se-Jin Park, Young-Bok Jung, Hwa-Jae Jung, Ho-Joong Jung, Hun Kyu Shin, Eugene Kim, et al. Outcome of Arthroscopic Single-Bundle Versus DoubleBundle Reconstruction of the Anterior Cruciate Ligament: A Preliminary 2-Year Prospective Study. Arthroscopy 2010; 26(5): 630-6.

6. Muneta T, Koga H, Morito T, Ogiuchi T, Yamamoto H, Shinomiya K. A retrospective study of the midterm outcome of two-bundle anterior cruciate ligament reconstruction using quadrupled semitendinosus tendon in comparison with one-bundle recon- struction. Arthroscopy 2006; 22:252-8.

7. Siebold R, Dehler C, Ellert T. Prospective randomized com- parison of double-bundle versus single-bundle anterior cruci- ate ligament reconstruction. Arthroscopy 2008 Feb; 24(2):137-45.

8. Odenstein M, Gillquist J. Functional anatomy of the anterior cruciate ligament and a rationale for reconstruction. J.Bone Joint Surg 1985; 67-A: 257-62.

9. Colombet P, Robinson J, Christel P. Morphology of anterior cruciate ligament attachments for anatomic reconstruction: a cadaveric dissection and radiographic study. Arthroscopy 2006; 22:984-92.

10. Morgan CD, Kalman VR, Grawl DM. Definitive land- marks for reproducible tibial tunnel placement in anterior cruciate ligament reconstruction. Arthroscopy 1995; 11:275-88.

11. Harner CD, Baek GH, Vogrin TM. Quantitative analysis of human cruciate ligament insertions. Arthroscopy 1999; $15: 741-9$.

12. Siebold R, Ellert T, Metz S. Femoral insertions of the anteromedial and posterolateral bundles of the

anterior cruciate ligament: morphometry and arthroscopic orientation models for double-bundle bone tunnel placement-a cadaver study. Arthroscopy 2008; 24:585-92.

13. Siebold R, Ellert T, Metz S. Tibial insertions of the anteromedial and posterolateral bundles of the anterior cruciate ligament: morphometry, arthroscopic landmarks, and orientation model for bone tunnel placement. Arthroscopy 2008; 24:154-61.

14. Kopf S. Musahl, S. Tashman, Szczodry M, Shen W, Fu FH. A systematic review of the femoral origin and tibial insertion morphology of the ACL Knee Surg Sports Traumatol Arthrosc 2009; 17:213-9.

15. Palmieri Bouchan RB, Hernández Téllez IE, García Valadez LR. Resultados funcionales en pacientes postoperados de reconstrucción artroscópica de ligamento cruzado anterior con técnica all-inside®. Rev Sanid Mil 2018; 72(2):110-7.

16. Jaramillo Quiceno GA, Orozco PD, Agudelo Zuluaga ID, Venencia AP, Castro Padilla JA. Reconstrucción anatómica del ligamento cruzado anterior. Técnica quirúrgica y reporte de casos. Rev Colomb Ortop Traumatol 2017; 31(2):63-7.

17. García JG, Chávez D, Vargas A, Díez M del P, Ruiz T. Valoración funcional en pacientes postoperados de reconstrucción de ligamento cruzado anterior. Acta Ortopédica Mex 2005; 19(2):67-74.

18. Arroyo-Aparicio JY, Cota GP, Hernandez ARM, Romero DLR, Ramirez JEV, Romero HV, et al. Functional Results after Anterior Cruciate Ligament Reconstruction with Isquiotibial Graft: Analytic Study of Two Kinds of Graft. ARC J Surg 2018; 4(3):11-8.

19. Gigante DF. Resultados de la Cirugía de Revisión del Ligamento Cruzado Anterior. Comparación con la Cirugía Primaria. 2011; 18:9.

20. Vilchez-Cavazos F, Dávila-Martínez A, Garza-Castro S de la, Simental-Mendía M, Garay-Mendoza D, Tamez-Mata Y, et al. Anterior cruciate ligament injuries treated with quadriceps tendon autograft versus hamstring autograft: A randomized controlled trial. Cir Cir 2020; 88(1):76-81.

21. Beynnon BD, Johnson RJ, Fleming BC, Kannus P, Kaplan M, Samani J, et al. Anterior Cruciate Ligament Replacement: Comparison of Bone-Patellar Tendon-Bone Grafts with Two-Strand Hamstring Grafts: A Prospective, Randomized Study. JBJS 2002; 84(9):1503-13. 